\title{
Review of: "Regulating expression of mistranslating tRNAs by readthrough RNA polymerase II transcription"
}

\author{
David JHF Knapp ${ }^{1}$ \\ 1 Université de Montréal
}

Potential competing interests: The author(s) declared that no potential competing interests exist.

In this manuscript Berg et al. develop and characterize a system for tuning the expression of the tRNA polIII promoter in Saccharomyces cerevisiae. They apply this system to express otherwise toxic tRNA variants which replace Proline amino acids with Serine and measure the effects of this mistranslation on cell growth and peptide composition. Their system appears to function, reversing growth defects caused by expression of toxic tRNA variants, and mitigating the mistranslation when the pol-II promoter is active. In addition to the direct effects of titrating mistranslation rates caused by variant tRNA, this system could be adapted to the tuning of guide RNA expression for CRISPR/Cas systems, though this has not been explored in the present work. The presented system could thus be of interest to researchers across a range of fields. While interesting, there are a number of changes which would improve the interpretability and robustness of the presented system and results:

Much of the data in the paper relies on the measure of doubling time based on growth curve fits. The example growth curves displayed, however, aren't displaying the individual data points, but only the fit curve. Interpretation of the fits would be substantially aided by showing the points along with the fit, as well as a measure of goodness-of-fit (several are automatically calculated in the R package used for curve fitting). As the core of this paper relies on the growth curve fits, they should be displayed for all doubling time data points throughout the manuscript (in a supplemental figure). There are also several cases where example curves are shown but not doubling time estimates (Figure 2C, Figure 4), and even one (Figure 6C) where no fit is performed and only a single data point displayed at each time. These examples leave it impossible to assess experimental variability or even whether more than one replicate has been performed.

There are several additional characterizations of the system itself which would help others interested in using it. Given that the system relies on opposing transcriptional pressures (pol-II in reverse, pol-III forward), a measure of expression levels from each promoter at varying levels of pol-II induction would facilitate the wider use of the system. Testing distances between the pol-II promoter and tRNA longer than $300 \mathrm{bp}$ (the maximum used in the manuscript) to determine how far the promoter can be before it loses repressive ability would also be extremely useful. Finally, a characterization of how the repression is influenced by the flanking sequence would be necessary to ensure that others who wish to apply the 
system could do so successfully. This is highlighted by the authors statement that “...we found that the native sequences flanking tRNA Ala did not permit repression..." suggesting that sequence context is important for system function.

For the statistics throughout the paper, multiple tests have been performed, however, no multiple testing correction is reported. Additionally, pair-wise testing would be of interest in several scenarios which has not been done but would allow better comparison between conditions.

Annotated plasmid sequences should be added if they're not being deposited on Addgene.

Page 3 - "Our goal was to engineer an inducible system to regulated..." should be "regulate" With regards to the statement around Figure S3 “...promoter decreases linearly with increasing concentrations...", it's difficult to say with the 3 points whether this is linear or a gradual exponential decay. it would be more accurate to say in a dose dependent manner (unless of course more doses are included and a linear fit shown to outperform others)

In discussion of Figure $6 \mathrm{~A} / \mathrm{S} 5$ it is stated that the cells are non-viable, though PI stain and their other results suggest that they're arrested rather than dead. This should be reconciled. Further adding to the confusion, in Figure $6 \mathrm{~B}, 10$ hours or more after dox addition the $1 \mathrm{~N}$ peak appears to start gaining sub $1 \mathrm{~N}$ copies. This would suggest some degree of cell death. Also, a representative image is shown but no indication of the total $\mathrm{N}$, nor a quantification. 